\title{
NONHOLONOMIC CONSTRAINTS: A NEW VIEWPOINT
}

\author{
J. GRABOWSKI, M. DE LEÓN, J. C. MARRERO, AND D. MARTÍN DE DIEGO
}

\begin{abstract}
The purpose of this paper is to show that, at least for Lagrangians of mechanical type, nonholonomic Euler-Lagrange equations for a nonholonomic linear constraint $D$ may be viewed as non-constrained Euler-Lagrange equations but on a new (generally not Lie) algebroid structure on $D$. The proposed novel formalism allows us to treat in a unified way a variety of situations in nonholonomic mechanics and gives rise to a version of Neoether Theorem producing actual first integrals in case of symmetries.
\end{abstract}

\section{Contents}

1. Introduction

2. Geometric Mechanics on general algebroids

3. Nonholonomic mechanics

4. The nonholonomic reduction of algebroids

5. Nonholonomic systems of mechanical type

6. Examples

References

References

\section{INTRODUCTION}

There are many approaches to geometric mechanics in the literature. We will work with a natural generalization of the framework for studying mechanical systems proposed by W. M. Tulczyjew [29, 30] (see also [32] and references therein). In the simplest form, the phase dynamics of the system is understand as the lagrangian submanifold $\Gamma$ of the symplectic manifold $\left(\mathrm{TT}^{*} M, \mathrm{~d}_{\mathrm{T}} \omega_{M}\right)$ equipped with tangent lift $\mathrm{d}_{\mathrm{T}} \omega_{M}$ of the canonical symplectic form $\omega_{M}$ of $\mathrm{T}^{*} M$. Here, $M$ represents the configuration manifold of the system and $\Gamma$ is obtained from the lagrangian submanifold $\mathrm{d} L(M) \subset \mathrm{T}^{*} \mathrm{~T} M$ induced by the Lagrangian $L: \mathrm{T} M \rightarrow \mathbb{R}$ via the canonical isomorphism $\varepsilon_{M}: \mathrm{T}^{*} \mathrm{~T} M \rightarrow \mathrm{TT}^{*} M$. In other words, the phase dynamics, as well as the Euler-Lagrange equations, are obtained in a simple way by means of the Tulczyjew differential $\Lambda_{L}=\varepsilon_{M} \circ \mathrm{d} L: M \rightarrow \mathrm{TT}^{*} M$. It is important to observe that both $\mathrm{TT}^{*} M$ and $\mathrm{T}^{*} \mathrm{~T} M$ are double vector bundles over $\mathrm{T}^{*} M$ and TM (see [12] and references therein). The resulting submanifold $\Gamma$ of TT* $M$ is a particular case of modelling dynamical systems as implicit differential equations defined by differential inclusions (see [23, 24]).

This framework admits an immediate generalization for more general morphisms $\varepsilon: \mathrm{T}^{*} E \rightarrow \mathrm{T} E^{*}$ of canonical double vector bundles associated with a vector bundle $E$ (see [9, 8]), inducing the Tulczyjew differentials $\Lambda_{L}=\varepsilon \circ \mathrm{d} L: M \rightarrow \mathrm{T} E^{*}$. This generalization includes as a particular case a theory of mechanical systems based on Lie algebroids, as proposed by A. Weinstein [36] and developed by many authors, however in a different geometrical setting (see, for instance, [6, 7, 19, 20, 25, 26]). The

2000 Mathematics Subject Classification. 70F25, 53D17, 70G45, 17B66, 70H03, $70 \mathrm{H} 45$.

Key words and phrases. General algebroids, Lie algebroids, double vector bundle, Nonholonomic mechanics, Lagrange-d'Alembert's equations, nonholonomic bracket.

This work has been partially supported by the Polish Ministry of Science and Higher Education under the grant No. N201 005 31/0115, MEC (Spain) Grants MTM 2006-03322, MTM 2007-62478, project Ingenio Mathematica (i-MATH) No. CSD 2006-00032 (Consolider-Ingenio 2010) and S-0505/ESP/0158 of the CAM. 
motivation for study systems on Lie algebroids is that they often appear naturally as results of some reduction procedures. This is is a situation similar to the one known in the theory of Hamiltonian systems: reductions may lead from a symplectic to a Poisson structure.

An additional challenge and one of the most fascinating topics in geometric mechanics is the study of constraints in this context. Of course, a general problem of putting constraints for the system in a variational setting involves constraints for velocities as well as constraints for virtual displacements, as was noticed already in [31. In some cases, however, one assumes that the constraints can be determined from a constraint subset $D$ of T $M$ (or, of $E$ in the algebroid case) by certain well-described procedures. The best known approaches of this type refer to the so called vakonomic and nonholonomic constraints. In the simplest situation, for $D$ being a linear nonholonomic constraint, i.e. just a vector subbundle of TM (or, of $E$ in the algebroid context), this procedure describes the nonholonomic EulerLagrange equations by means of the d'Alembert principle, having analogs also in the algebroid case [7, 18, 27, 8]. We should stress that our nonholonomic constraints are linear in the broader sense, i.e. they are subbundles over submanifolds of the original base manifold. The nonholonomic EulerLagrange equations are commonly viewed as being not variational equations. In [8] it has been pointed out that it is not exactly the case, if we extend slightly our understanding of Variational Calculus.

In this paper we continue studying nonholonomic constraints on algebroids and showing that, at least for Lagrangians of mechanical type, the nonholonomic Euler-Lagrange equations are just nonconstrained Euler-Lagrange equations but for a special algebroid structure on the constraint subbundle (see also [18). This shows that mechanical systems based on general (not necessary Lie) algebroids appear naturally in the presence of nonholonomic constraints and gives a powerful geometrical tool when dealing with constrained systems. In particular, we get a version of Noether Theorem with true first integrals for nonholonomic systems. We do not get all possible algebroids on $D$ applying our procedure. In particular, if the original structure was a Lie algebroid, then the new algebroid bracket is automatically skew- symmetric, so we deal with a quasi-Lie algebroid. One can the associate with the sequence of procedures, like reduction by symmetries and passing to a nonholonomic constraint, the sequence of the corresponding novel structures serving as appropriate geometrical tools in describing the systems:

$\mathrm{T} M \stackrel{\text { reduction by symmetries }}{\longrightarrow}$ Lie algebroid $\stackrel{\text { nonholonomic constraint }}{\longrightarrow}$ quasi-Lie algebroid.

All this is of course closely related to the discovery of the role of the nonholonomic quasi-Poisson brackets [22, 35, 15, 4], this time not in the Hamilton but in the Lagrange picture.

The paper is organized as follows. In the next section, we recall after 9 the basic ideas of developing mechanics on a general algebroid $E$, in particular, the Euler-Lagrange equations. Then, we construct in this setting an analog of the Tulczyjew differential for linear nonholonomic constraints, together with the corresponding nonholonomic Euler-Lagrange equations. In Section 4 we study reductions of a general algebroid to an algebroid on a nonholonomic constraint $D$ (satisfying a natural admissibility condition) along a given projection. We discuss also the problem, what algebroids can be obtained in this way, if we start with Lie algebroids.

Section 5 is the most important part of our paper. For Lagrangian functions $L$ of mechanical type ('kinetic energy - potential'), we show that the non-constrained Euler-Lagrange equations on $D$, derived for the reduced Lagrangian $l=L_{\mid D}$ and for the reduced algebroid structure on $D$ along the orthogonal projection associated with the kinetic energy, coincide with the nonholonomic EulerLagrange equations. Moreover, for this nonholonomic case, we can apply therefore the generalization of the Noether Theorem proved in [9] to obtain actual first integrals. Passing to the nonholonomic constraints does not requires therefore any change in our unified algebroid approach to mechanics: nonholonomic Euler-Lagrange equations are included in our framework.

We end up with two well-known examples of nonholonomic constraints, the Chaplygin sleigh and the snakebord, to show how simply the corresponding equations of motions can be derived by means of our method. 


\section{Geometric Mechanics on General algebroids}

Let $M$ be a smooth manifold and $\left(x^{i}\right), i=1, \ldots, n$, be a coordinate system in $M$. Denote by $\tau_{M}: \mathrm{T} M \rightarrow M$ the tangent vector bundle, with induced coordinates $\left(x^{i}, \dot{x}^{j}\right)$, and by $\pi_{M}: \mathrm{T}^{*} M \rightarrow M$ the contangent bundle, with induced coordinates $\left(x^{i}, p_{j}\right)$.

Let $\tau: E \rightarrow M$ be a vector bundle and $\pi: E^{*} \rightarrow M$ its dual bundle. Taking a local basis $\left\{e_{a}\right\}$ of sections of $E$, then we have the corresponding local coordinates $\left(x^{i}, y^{a}\right)$ on $E$, where $y^{a}(e)$ is the ath-coordinate of $e \in E$ in the given basis. We denote by $\left(x^{i}, \xi_{a}\right)$ the corresponding coordinates of the dual bundle $\pi: E^{*} \rightarrow M$. One can also say that $\xi_{a}$ is the fiber-wise linear local function $\iota\left(e_{a}\right)$ on $E^{*}$ corresponding to the local section $e_{a}$ of $E$. We have also adapted local coordinates:

$$
\begin{aligned}
& \left(x^{i}, y^{a}, \dot{x}^{j}, \dot{y}^{b}\right) \quad \text { in T } E, \quad\left(x^{i}, \xi_{a}, \dot{x}^{j}, \dot{\xi}_{b}\right) \text { in T } E^{*}, \\
& \left(x^{i}, y^{a}, p_{j}, \pi_{b}\right) \quad \text { in } \mathrm{T}^{*} E, \quad\left(x^{i}, \xi_{a}, p_{j}, \varphi^{b}\right) \quad \text { in } \mathrm{T}^{*} E^{*} \text {. }
\end{aligned}
$$

It is well known (cf. 16) that the cotangent bundles $\mathrm{T}^{*} E$ and $\mathrm{T}^{*} E^{*}$ are examples of double vector bundles:
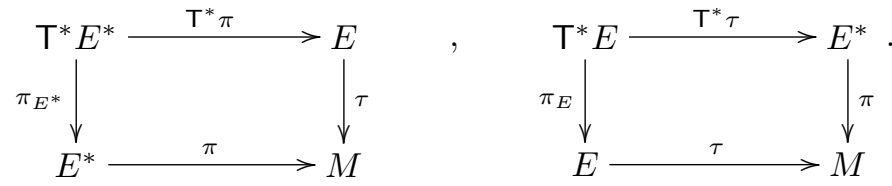

The concept of a double vector bundle is due to J. Pradines [33, 34, see also [21, 16]. In particular, all arrows correspond to vector bundle structures and all pairs of vertical and horizontal arrows are vector bundle morphisms. The double vector bundles have been recently characterized [10] in a simple way as two vector bundle structures whose Euler vector fields commute. The above double vector bundles are canonically isomorphic with the isomorphism

$$
\mathcal{R}_{\tau}: \mathrm{T}^{*} E \rightarrow \mathrm{T}^{*} E^{*}
$$

being simultaneously an anti-symplectomorphism (cf. [16, 12]). In adapted local coordinates, $\mathcal{R}_{\tau}$ is given by

$$
\mathcal{R}_{\tau}\left(x^{i}, y^{a}, p_{j}, \pi_{b}\right)=\left(x^{i}, \pi_{a},-p_{j}, y^{b}\right) .
$$

This means that we can identify $\pi_{b}$ and $\xi_{b}$, as well as $y^{b}$ with $\varphi^{b}$, so we will use local coordinates $(x, y, p, \xi)$ on $\mathrm{T}^{*} E$ and local coordinates $(x, \xi, p, y)$ on $\mathrm{T}^{*} E^{*}$ in full agreement with the isomorphism (2.1.

For describing the dynamics of a Lagrangian or Hamiltonian systems it is necessary to give an additional ingredient, typically, a Lie algebroid structure or, more generally, the algebroid structure. In several papers, many authors have studied Lie algebroid structures as a unified geometric framework, general enough to account for different mechanical systems (defined on tangent bundles, on Lie algebras, quotients by Lie groups actions, etc.).

It is well known that Lie algebroid structures on a vector bundle $E$ correspond to linear Poisson tensors on $E^{*}$. A 2-contravariant tensor $\Pi$ on $E^{*}$ is called linear if the corresponding mapping $\widetilde{\Pi}: \mathrm{T}^{*} E^{*} \rightarrow \mathrm{T} E^{*}$ induced by contraction is a morphism of double vector bundles. This is the same as to say that the corresponding bracket of functions is closed on (fiber-wise) linear functions. The commutative diagram

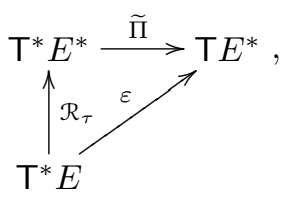

composed with 2.1), describes a one-to-one correspondence between linear 2-contravariant tensors $\Pi$ on $E^{*}$ and homomorphisms of double vector bundles (cf. [16, 12]) covering the identity on $E^{*}$ : 


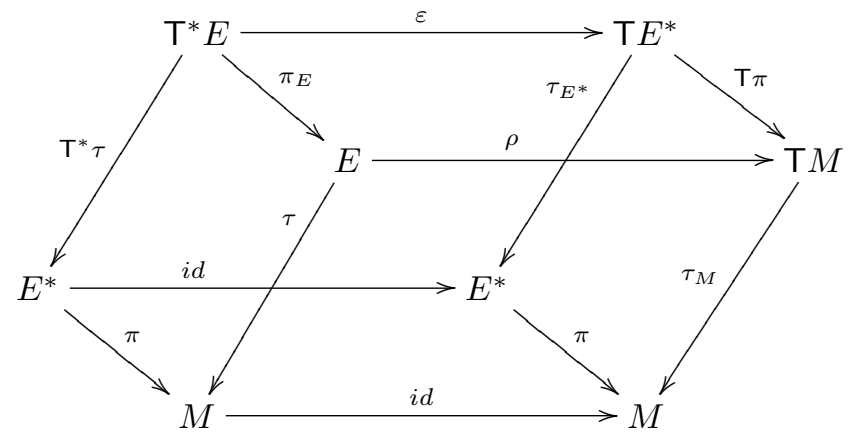

In local coordinates, every $\varepsilon$ as above is of the form

$$
\varepsilon\left(x^{i}, y^{a}, p_{j}, \pi_{b}\right)=\left(x^{i}, \pi_{a}, \sum_{c} \rho_{c}^{j}(x) y^{c}, \sum_{a, c} \mathrm{e}_{a b}^{c}(x) y^{a} \pi_{c}+\sum_{i} \sigma_{b}^{i}(x) p_{i}\right)
$$

and it corresponds to the linear tensor

$$
\Pi_{\varepsilon}=\sum_{a, b, c} \mathcal{C}_{a b}^{c}(x) \xi_{c} \partial_{\xi_{a}} \otimes \partial_{\xi_{b}}+\sum_{i, b} \rho_{b}^{i}(x) \partial_{\xi_{b}} \otimes \partial_{x^{i}}-\sum_{a, j} \sigma_{a}^{j}(x) \partial_{x^{j}} \otimes \partial_{\xi_{a}} .
$$

In [12] by algebroids we meant the morphisms 2.2 of double vector bundles covering the identity on $E^{*}$, while Lie algebroids were those algebroids for which the tensor $\Pi_{\varepsilon}$ is a Poisson tensor. We can consider the adjoint tensor $\Pi_{\varepsilon}^{+}$, i.e. the 2-contravariant tensor obtained from $\Pi_{\varepsilon}$ by transposition:

$$
\Pi_{\varepsilon}^{+}=\sum_{a, b, c} \mathcal{C}_{b a}^{c}(x) \xi_{c} \partial_{\xi_{a}} \otimes \partial_{\xi_{b}}+\sum_{i, b} \rho_{b}^{i}(x) \partial_{x^{i}} \otimes \partial_{\xi_{b}}-\sum_{a, j} \sigma_{a}^{j}(x) \partial_{\xi_{a}} \otimes \partial_{x^{j}}
$$

and the corresponding adjoint algebroid structure $\varepsilon^{+}$. Algebroids $\varepsilon$ corresponding to skew-symmetric $\Pi_{\varepsilon}$ (anti-symmetric brackets $[\cdot, \cdot]_{\varepsilon}$ ), i.e. such that $\varepsilon^{+}=-\varepsilon$, we will call quasi-Lie algebroids. The relation to the canonical definition of Lie algebroid is given by the following theorem (cf. [13, 12]).

Theorem 2.1. An algebroid structure $(E, \varepsilon)$ can be equivalently defined as a bilinear bracket $[\cdot, \cdot]_{\varepsilon}$ on sections of $\tau: E \rightarrow M$, together with vector bundle morphisms $\rho, \sigma: E \rightarrow \mathrm{T} M$ (left and right anchors), such that

$$
[f X, g Y]_{\varepsilon}=f \rho(X)(g) Y-g \sigma(Y)(f) X+f g[X, Y]_{\varepsilon}
$$

for $f, g \in \mathrm{e}^{\infty}(M), X, Y \in \operatorname{Sec}(E)$. The bracket and anchors are related to the 2-contravariant tensor $\Pi_{\varepsilon}$ by the formulae

$$
\begin{aligned}
\iota\left([X, Y]_{\varepsilon}\right) & =\{\iota(X), \iota(Y)\}_{\Pi_{\varepsilon}}, \\
\pi^{*}(\rho(X)(f)) & =\left\{\iota(X), \pi^{*} f\right\}_{\Pi_{\varepsilon}}, \\
\pi^{*}(\sigma(X)(f)) & =\left\{\pi^{*} f, \iota(X)\right\}_{\Pi_{\varepsilon}} .
\end{aligned}
$$

The algebroid $(E, \varepsilon)$ is a quasi-Lie algebroid if and only if the tensor $\Pi_{\varepsilon}$ is skew-symmetric and it is a Lie algebroid if and only if the tensor $\Pi_{\varepsilon}$ is a Poisson tensor.

The canonical example of a mapping $\varepsilon$ in the case of $E=\mathrm{T} M$ is given by $\varepsilon=\varepsilon_{M}=\alpha_{M}^{-1}$ - the inverse to the Tulczyjew isomorphism $\alpha_{M}: \mathrm{TT}^{*} M \rightarrow \mathrm{T}^{*} \mathrm{~T} M$. In general, the algebroid structure map $\varepsilon$ is not an isomorphism and, consequently, its dual $\kappa=\varepsilon^{*}$ with respect to the right projection is a relation and not a mapping. Some authors (see [19] for the case of Lie algebroids) have introduced the concept of prolongation of a Lie algebroid to maintain some of the original properties of the Tulzcyjew mapping (isomorphism and symplecticity), but, for the purposes of this paper, it is not necessary to use this formalism. 
The double vector bundle morphism $(2.2)$ can be extended to the following algebroid analogue of the so called Tulczyjew triple

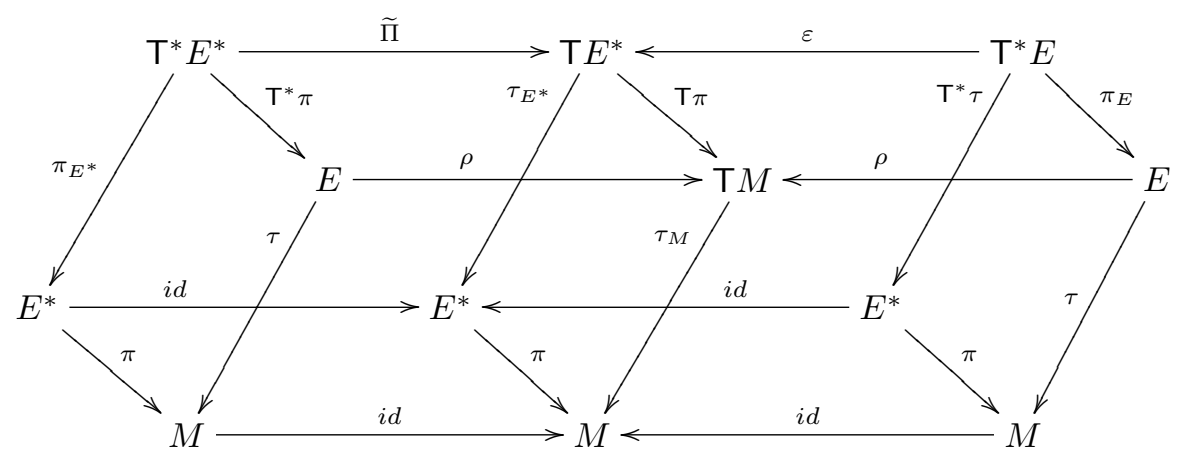

The left-hand side is Hamiltonian, the right-hand side is Lagrangian, and the dynamics lives in the middle.

We introduce now the dynamics through a Lagrangian $L: E \rightarrow \mathbb{R}$ which defines two smooth maps: the Legendre mapping: $\lambda_{L}: E \rightarrow E^{*}, \lambda_{L}=\tau_{E^{*}} \circ \varepsilon \circ \mathrm{d} L$, which is covered by the Tulczyjew differential $\Lambda_{L}: E \rightarrow \mathrm{T} E^{*}, \Lambda_{L}=\varepsilon \circ \mathrm{d} L:$

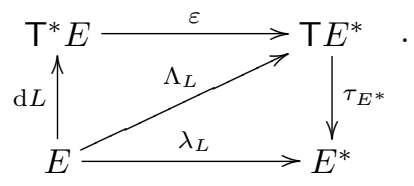

The lagrangian function defines the phase dynamics $\Gamma_{L}=\Lambda_{L}(E) \subset \mathrm{T} E^{*}$ which can be understood as an implicit differential equation on $E^{*}$, solutions of which are 'phase trajectories' of the system $\beta: \mathbb{R} \rightarrow E^{*}$ and satisfy $\mathbf{t} \beta(t) \in \Gamma_{L}$, where $\mathbf{t} \beta$ is the tangent prolongation of $\beta, \mathbf{t} \beta(t)=(\beta(t), \dot{\beta}(t))$. An analog of the Euler-Lagrange equations for curves $\gamma: \mathbb{R} \rightarrow E$ is in turn:

$$
\left(E_{L}\right) \quad \mathbf{t}\left(\lambda_{L} \circ \gamma\right)=\Lambda_{L} \circ \gamma .
$$

In local coordinates, $\Gamma_{L}$ has the parametrization by $\left(x^{i}, y^{a}\right)$ via $\Lambda_{L}$ in the form (cf. 2.3p)

$$
\Lambda_{L}\left(x^{i}, y^{a}\right)=\left(x^{a}, \frac{\partial L}{\partial y^{a}}(x, y), \sum_{c} \rho_{c}^{j}(x) y^{c}, \sum_{a, c} \mathrm{e}_{a b}^{c}(x) y^{a} \frac{\partial L}{\partial y^{c}}(x, y)+\sum_{i} \sigma_{b}^{i}(x) \frac{\partial L}{\partial x^{i}}(x, y)\right)
$$

and the equation $\left(E_{L}\right)$, for $\gamma(t)=\left(x^{i}(t), y^{a}(t)\right)$, reads

$$
\left(E_{L}\right): \quad \frac{\mathrm{d} x^{i}}{\mathrm{~d} t}=\sum_{c} \rho_{c}^{i}(x) y^{c}, \quad \frac{\mathrm{d}}{\mathrm{d} t}\left(\frac{\partial L}{\partial y^{b}}\right)=\sum_{a, c} \mathcal{C}_{a b}^{c}(x) y^{a} \frac{\partial L}{\partial y^{c}}(x, y)+\sum_{i} \sigma_{b}^{i}(x) \frac{\partial L}{\partial x^{i}}(x, y) .
$$

As one can see from (2.7), the solutions are automatically admissible curves in $E$, i.e. the velocity $\mathbf{t}(\tau \circ \gamma)(t)$ is $\rho(\gamma(t))$.

With this framework it is possible to write in a unified point of view many equations of different mechanical systems that usually, in the literature, appears as different ones (Classical Euler-Lagrange equations, Lagrange-Poincaré equations after reduction by the action of a Lie group, Euler-Poincaré equations, etc.). (See [6, 19] for applications of the theory in the case when $(E, \varepsilon)$ is a Lie algebroid).

\section{NONHOLONOMIC MECHANICS}

We can start from a general Lie algebroid $(E, \varepsilon)$ keeping in mind the standard case $E=\mathbf{T} M$. A (linear) nonholonomic Lagrangian system is determined by a Lagrangian function $L: E \rightarrow \mathbb{R}$ and a vector subbundle $D, \operatorname{rank} D=n-r$, of the bundle $E$. We will accept subbundles over a submanifold, so let us denote $D_{M}=\tau(D)$. By $i_{D}: D \hookrightarrow E$ let us denote the inclusion and by $i_{D}^{*}: E_{\mid D_{M}}^{*} \rightarrow D^{*}$ the dual map.

Because the solutions of the dynamics in $E$ should be admissible curves, we need an admissibility condition ensuring that there are admissible curves through every point of $D$. The natural condition we 
take is $\rho(D) \subset \mathbf{T} D_{M}$. By strong admissibility condition we will mean that $D$ satisfies the integrabilty condition with respect to both algebroid structures: $\varepsilon$ and $\varepsilon^{+}$, i.e.

$$
\rho(D) \subset \mathbf{T} D_{M} \text { and } \sigma(D) \subset \mathbf{T} D_{M} .
$$

The Lagrangian function $L: E \rightarrow \mathbb{R}$ and the vector subbundle $D$ define also the smooth map - the constrained Tulczyjew differential

$$
\Lambda_{L}^{D}: D \rightarrow \mathbf{T} D^{*}, \quad \Lambda_{L}^{D}=\mathbf{T} i_{D}^{*} \circ \varepsilon \circ \mathrm{d} L
$$

covering the constrained Legendre map

$$
\lambda_{L}^{D}: D \rightarrow D^{*}, \quad \lambda_{L}^{D}=i_{D}^{*} \circ \lambda_{L} .
$$

The diagram picture is the following

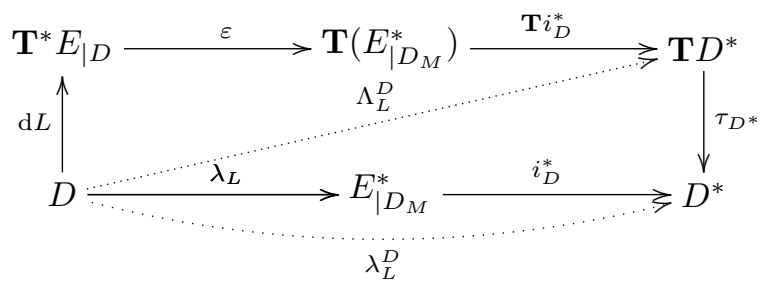

Definition 3.1. The bundle $D^{*}$ we call the phase space of the nonholonomic system and the implicit differential equation $\Gamma_{L}^{D}=\Lambda_{L}^{D}(D)$, being a subset of $\mathbf{T} D^{*}$, we interpret as the phase dynamics of the nonholonomic system. A curve $\gamma: I \rightarrow D$ is a solution of the nonholonomic Euler-Lagrange equation if and only if $\Lambda_{L}^{D} \circ \gamma$ is an admissible curve in $\mathbf{T} D^{*}$, i.e. the nonholonomic Euler-Lagrange equation reads

$$
\left(n E_{L}\right): \quad \mathbf{t}\left(\lambda_{L}^{D} \circ \gamma\right)=\Lambda_{L}^{D} \circ \gamma
$$

To find the explicit form of the nonholonomic Euler-Lagrange equation (3.5), consider local coordinates $\left(x^{I}\right)=\left(x^{i}, x^{\iota}\right)$ on a open set $U$ of $M$ such that $D_{M}$ is determined by the constraint $x^{\iota}=0$. A local basis $\left\{e_{a}\right\}_{a=1, \ldots, n-r}$ of sections of $D$ we can extend to local sections of $E$ and complete them to a local basis of sections $\left\{e_{a}, e_{\alpha}\right\}$ of the vector bundle $E$. Then, in coordinates $\left(x^{I}, y^{A}\right)=\left(x^{i}, x^{\iota}, y^{a}, y^{\alpha}\right)$ adapted to this bases, the local equations defining the constrained subbundle $D$ as a vector subbundle of $E$ over $D_{M}$ are $x^{\iota}=0, y^{\alpha}=0$. Note that admissibility of the constraint $D$ means that $\rho_{a}^{\iota}\left(x^{i}, 0\right)=0$.

Taking local coordinates $\left(x^{i}, y^{a}\right)$ on $D$, we may write then $i_{D}: D \hookrightarrow E$ as $i_{D}\left(x^{i}, y^{a}\right)=\left(x^{i}, 0, y^{a}, 0\right)$ and $i_{D}^{*}\left(x^{i}, 0, \xi_{a}, \xi_{\alpha}\right)=\left(x^{i}, \xi_{a}\right)$, so

$$
\mathbf{T} i_{D}^{*}\left(x^{i}, 0, \xi_{A}, \dot{x}^{j}, 0, \dot{\xi}_{B}\right)=\left(x^{i}, \xi_{a}, \dot{x}^{j}, \dot{\xi}_{b}\right) .
$$

For the adapted local coordinates $\left(x^{i}, x^{\iota}, y^{a}, y^{\alpha}, p_{j}, p_{\gamma}, \xi_{b}, \xi_{\beta}\right)$ in $\mathbf{T}^{*} E$, the map $\varepsilon$ reduced to $\left(\mathbf{T}^{*} E\right)_{\mid D}$ takes values in $\mathbf{T}\left(E_{\mid D_{M}}^{*}\right)$ (admissibility) and reads

$$
\varepsilon\left(x^{i}, 0, y^{a}, 0, p_{J}, \xi_{B}\right)=\left(x^{i}, 0, \xi_{A}, \rho_{d}^{j}\left(x^{i}, 0\right) y^{d}, 0, \varrho_{d B}^{C}\left(x^{i}, 0\right) y^{d} \xi_{C}+\sigma_{B}^{J}\left(x^{i}, 0\right) p_{J}\right) .
$$

Therefore

$$
\mathbf{T} i_{D}^{*} \circ \varepsilon\left(x^{i}, 0, y^{a}, 0, p_{J}, \xi_{B}\right)=\left(x^{i}, \xi_{a}, \rho_{d}^{j}\left(x^{i}, 0\right) y^{d}, \mathcal{C}_{d b}^{C}\left(x^{i}, 0\right) y^{d} \xi_{C}+\sigma_{b}^{J}\left(x^{i}, 0\right) p_{J}\right)
$$

and

$$
\begin{aligned}
& \Lambda_{L}^{D}\left(x^{i}, y^{a}\right) \\
& =T i_{D}^{*}\left(\varepsilon\left(x^{i}, 0, y^{a}, 0, \frac{\partial L}{\partial x^{J}}\left(x^{i}, 0, y^{a}, 0\right), \frac{\partial L}{\partial y^{B}}\left(x^{i}, 0, y^{a}, 0\right)\right)\right. \\
& =\left(x^{i}, \frac{\partial L}{\partial y^{b}}\left(x^{i}, 0, y^{a}, 0\right), \rho_{d}^{j}\left(x^{i}, 0\right) y^{d}, \mathrm{C}_{d b}^{C}\left(x^{i}, 0\right) y^{d} \frac{\partial L}{\partial y^{C}}\left(x^{i}, 0, y^{a}, 0\right)+\sigma_{b}^{J}\left(x^{i}, 0\right) \frac{\partial L}{\partial x^{J}}\left(x^{i}, 0, y^{a}, 0\right)\right)
\end{aligned}
$$

Therefore, locally, the nonholonomic Euler-Lagrange equations read:

$$
\begin{gathered}
x^{\iota}=0, \quad y^{\alpha}=0, \quad \frac{d x^{j}}{d t}=\rho_{d}^{j}\left(x^{i}, 0\right) y^{d}, \\
\left.\frac{d}{d t}\left(\frac{\partial L}{\partial y^{b}}\left(x^{i}, 0, y^{a}, 0\right)\right)=\mathcal{C}_{d b}^{C}\left(x^{i}, 0\right) y^{d} \frac{\partial L}{\partial y^{C}}\left(x^{i}, 0, y^{a}, 0\right)+\sigma_{b}^{J}\left(x^{i}, 0\right) \frac{\partial L}{\partial x^{J}}\left(x^{i}, 0, y^{a}, 0\right)\right) .
\end{gathered}
$$


In the case of a Lie algebroid $\sigma_{A}^{i}=\rho_{A}^{i}$, and if the subbundle $D$ is over the whole base manifold $M, D_{M}=M$, the previous equations are precisely the nonholonomic equations obtained in [7] (see Equations 3.8).

\section{The nonholonomic Reduction of Algebroids}

It has been recognized a long time ago 22, 35] that nonholonomic constraints may lead to certain nonholonomic brackets that do not satisfy the Jacobi identity. We will show that linear nonholonomic systems of mechanical type on general algebroids are again systems on general algebroids. But even if we start with a Lie algebroid, the new algebroid is, in general, no longer a Lie algebroid but certain quasi-Lie algebroid associated with a linear bi-vector field. This observation, made already in 8 (see also [18]), puts new light to the role of quasi-Poisson brackets, i.e. the brackets represented by arbitrary bi-vector fields and not satisfying, in general, the Jacobi identity (see [8, 18). This shows, on the other hand, that developing Mechanics on general algebroids makes sense, as the reduction to a nonholonomic constraint will move us, in general, from the Lie algebroid picture into a more general one.

Let $\varepsilon$ be an algebroid structure on a vector bundle $E$ over $M$ associated with the tensor $\Pi_{\varepsilon}$ with the local form 2.4. For a linear subbundle $D$ in $E$ over a submanifold $D_{M} \subset M$, satisfying the strong admissibility condition (3.1), consider a decomposition

$$
E_{\mid D_{M}}=D \oplus_{D_{M}} D^{\perp}
$$

and the associated projection $P: E_{\mid D_{M}} \rightarrow D$. We can construct the morphism $\varepsilon_{P}: \mathbf{T}^{*} D \rightarrow \mathbf{T} D^{*}$ of double vector bundles as follows.

To have the corresponding expressions in local coordinates, consider local coordinates $\left(x^{I}\right)=\left(x^{i}, x^{\iota}\right)$ on a open set $U$ of $M$ such that $D_{M}$ is determined by the constraint $x^{\iota}=0$. Local bases $\left\{e_{a}\right\}_{a=1, \ldots, n-r}$ and $\left\{e_{\alpha}\right\}_{\alpha=n-r+1, \ldots, n}$ of sections of $D$ and $D^{\perp}$, respectively, can be extended to a basis $e_{A}$ of local sections of $E$. Then, we get the coordinates $\left(x^{I}, y^{A}\right)=\left(x^{i}, x^{\iota}, y^{a}, y^{\alpha}\right)$ adapted to these bases and the adapted coordinates $\left(x^{I}, y^{A}, p_{J}, \xi_{B}\right)=\left(x^{i}, x^{\iota}, y^{a}, y^{\alpha}, p_{j}, p_{\gamma}, \xi_{b}, \xi_{\beta}\right)$ in $\mathbf{T}^{*} E$. We get automatically coordinates $\left(x^{i}, y^{a}\right)$ in $D$ and $\left(x^{i}, y^{a}, y^{\alpha}\right)$ in $E_{\mid D_{M}}$, and the adapted coordinates $\left(x^{i}, y^{a}, p_{j}, \xi_{a}\right)$ and $\left(x^{i}, y^{A}, p_{j}, \xi_{B}\right)$ in $\mathbf{T}^{*} D$ and $\mathbf{T}^{*}\left(E_{\mid D_{M}}\right)$, respectively.

Let us consider the phase lift $\mathbf{T}^{*} P: \mathbf{T}^{*} D \longrightarrow \mathbf{T}^{*}\left(E_{\mid D_{M}}\right)$ which, as often happens which phase lifts, is not a map but only a relation. In our local coordinates,

$$
\mathbf{T}^{*} P\left(x^{i}, y^{a}, p_{j}, \xi_{b}\right)=\left(x^{i}, y^{A}, p_{j}, \xi_{b}, 0\right) .
$$

We have also the embedding $i_{E_{\mid D_{M}}}: E_{\mid D_{M}} \rightarrow E$ whose phase lift, restricted to $\mathbf{T}^{*} E_{\mid D}$,

$$
\mathbf{T}^{*}\left(i_{E_{\mid D_{M}}}\right): \mathbf{T}^{*} E_{\mid D} \longrightarrow \mathbf{T}^{*}\left(E_{\mid D_{M}}\right)
$$

is the relation

$$
\mathbf{T}^{*}\left(i_{E_{\mid D_{M}}}\right)\left(x^{i}, 0, y^{a}, 0, p_{J}, \xi_{B}\right)=\left(x^{i}, y^{A}, p_{j}, \xi_{B}\right) .
$$

The composition of relations

$$
\mathbf{T}^{*}\left(i_{E_{\mid D_{M}}}\right)^{-1} \circ \mathbf{T}^{*} P: \mathbf{T}^{*} D \longrightarrow \mathbf{T}^{*} E_{\mid D}
$$

has the local form

$$
\left(\mathbf{T}^{*}\left(i_{E_{\mid D_{M}}}\right)^{-1} \circ \mathbf{T}^{*} P\right)\left(x^{i}, y^{a}, p_{j}, \xi_{b}\right)=\left(x^{i}, 0, y^{a}, 0, p_{J}, \xi_{b}, 0\right) .
$$

But, as $\sigma_{b}^{\iota}\left(x^{i}, 0\right)=0$ (strong admissibility condition), the value of $\mathbf{T} i_{D}^{*} \circ \varepsilon$ in 3.6 does not depend on $p_{\gamma}$ and the composition

$$
\varepsilon_{P}=\mathbf{T} i_{D}^{*} \circ \varepsilon \circ \mathbf{T}^{*}\left(i_{E_{\mid D_{M}}}\right)^{-1} \circ \mathbf{T}^{*} P: \mathbf{T}^{*} D \rightarrow \mathbf{T} D^{*}
$$

is a well-defined map which, in local coordinates, reads

$$
\varepsilon_{P}\left(x^{i}, y^{a}, p_{j}, \xi_{b}\right)=\left(x^{i}, \xi_{a}, \rho_{d}^{j}\left(x^{i}, 0\right) y^{d}, \mathrm{C}_{d b}^{c}\left(x^{i}, 0\right) y^{d} \xi_{c}+\sigma_{b}^{k}\left(x^{i}, 0\right) p_{k}\right) .
$$

This is of course an algebroid structure on the bundle $D$. This algebroid structure can be described in a more straightforward way as follows. 
The decomposition 4.1 gives the dual decomposition

$$
E_{\mid D_{M}}^{*}=D^{*} \oplus_{D_{M}}\left(D^{\perp}\right)^{*}
$$

and the corresponding projection $P^{*}: E_{\mid D_{M}}^{*} \rightarrow D^{*}$. This projection does not give us a canonical projection of vectors and, more generally, contravariant tensors on $E$ at points of $E_{\mid D_{M}}^{*}$, unless they are tangent to $E_{\mid D_{M}}^{*}$. But the tensor $\Pi_{\varepsilon}$ with the local form 2.4 is a sum of tensor product with at least one part in the product being vertical. Moreover, due to strong admissibility condition for $D$, there is a unique decomposition $\Pi_{\varepsilon}=\Pi_{\varepsilon_{D}}+\Pi_{\varepsilon_{D^{\perp}}}$, where $\Pi_{\varepsilon_{D}}$ is a linear tensor tangent to $D^{*}$ and the vertical parts of $\Pi_{\varepsilon_{D^{\perp}}}$ are tangent to $\left(D^{\perp}\right)^{*}$, so they will be killed by the projection, independently how the other part of the product is. This gives a well-defined projection $\mathbf{T}\left(P^{*}\right)\left(\Pi_{\varepsilon}\right)=\Pi_{\varepsilon_{P}}$, when we restrict to the points of $D^{*}$.

To put it differently, we can take any smooth projection $\mathcal{P}$ from a neighbourhood of $E_{\mid D_{M}}^{*}$ onto $E_{\mid D_{M}}^{*}$. Then,

$$
\mathbf{T}\left(P^{*}\right)\left(\Pi_{\varepsilon}\right):=\mathbf{T}\left(P^{*} \circ \mathcal{P}\right)\left(\Pi_{\varepsilon}\right)
$$

is a linear 2-contravariant tensor on $D$ which does not depend on the choice of $\mathcal{P}$. This tensor defines on $D^{*}$ the nonholonomic bracket $\{\cdot, \cdot\}_{\varepsilon_{P}}$ associated with the projection $P$.

On the level of the algebroid bracket $[\cdot, \cdot]_{\varepsilon}$ this procedure is the following. The strong admissibility condition for $D$ implies that the bracket $[X, Y]_{\varepsilon}$ of sections of $D$ is a section of $E_{\mid D_{M}}$. Projecting this section to $D$ along $P$ gives us a bracket

$$
[X, Y]_{\varepsilon_{P}}=P[X, Y]_{\varepsilon}
$$

on sections of $D$ - the nonholonomic restriction of $[\cdot, \cdot]$ along $P$. This is an algebroid bracket with the original anchors.

Of course, if the constraint bundle $D$ is a subalgebroid of $E$, then it is a holonomic constraint and the projection $P$ plays no role: the tensor $\Pi_{\varepsilon}$ is tangent to $D$ and we just take the restriction. It is however clear that, in the true nonholonomic case, the nonholonomic bracket need not satisfy the Jacobi identity, even when the original bracket does. In local coordinates,

$$
\Pi_{\varepsilon_{P}}=\sum_{a, b, c} \mathfrak{C}_{a b}^{c}\left(x^{i}, 0\right) \xi_{c} \partial_{\xi_{a}} \otimes \partial_{\xi_{b}}+\sum_{j, b} \rho_{b}^{j}\left(x^{i}, 0\right) \partial_{\xi_{b}} \otimes \partial_{x^{j}}-\sum_{a, j} \sigma_{a}^{j}\left(x^{i}, 0\right) \partial_{x^{j}} \otimes \partial_{\xi_{a}} .
$$

and the corresponding nonholonomic bracket reads:

$$
\begin{aligned}
\left\{\xi_{a}, \xi_{b}\right\}_{\varepsilon_{P}} & =\mathcal{C}_{a b}^{c}\left(x^{i}, 0\right) \xi_{c}, \\
\left\{\xi_{b}, x^{j}\right\}_{\varepsilon_{P}} & =\rho_{b}^{j}\left(x^{i}, 0\right), \\
\left\{x^{j}, \xi_{a}\right\}_{\varepsilon_{P}} & =-\sigma_{a}^{j}\left(x^{i}, 0\right), \\
\left\{x^{k}, x^{j}\right\}_{\varepsilon_{P}} & =0 .
\end{aligned}
$$

In the case of a Lie algebroid, we have $\mathcal{C}_{a b}^{c}=-\bigodot_{b a}^{c}, \sigma_{A}^{j}=\rho_{A}^{j}$, and the above bracket corresponds to the one introduced by [35] (see also [7] and references therein) for the subbundles over the total base $M$.

A natural question arises here: what Lie algebroid structures we can obtain as nonholonomic restrictions of Lie algebroid brackets? Of course, as the brackets must be skew-symmetric automatically, the algebroids must be necessarily quasi-Lie algebroids. We will call them nonholonomic quasi-Lie algebroids. In other words: which linear bi-vector fields on a vector bundle $D \rightarrow M$ can be obtained by projections of Poisson tensors from a bigger vector bundle $E \rightarrow M$. For nonholonomic quasi-Lie algebras the answer is simple.

Theorem 4.1. Any quasi-Lie algebroid D with the trivial anchor is a nonholonomic quasi-Lie algebroid.

Proof. As the anchor is trivial, we can clearly reduce to finite-dimensional quasi-Lie algebras. Take a basis $e_{i}$ of $D$ and the corresponding linear coordinates $y^{i}, i=1, \ldots, n$. The algebroid bracket in $D$ is then determined by the structure constants $c_{i j}^{k}$,

$$
\left[e_{i}, e_{j}\right]=c_{i j}^{k} e_{k}
$$


satisfying $c_{i j}^{k}=-c_{j i}^{k}$. Let us consider a new algebra $E$ with the basis $e_{i}, f_{j}, i=1, \ldots, n$ and the bracket $[\cdot, \cdot]_{E}$ for which $f_{j}$ are central elements and

$$
\left[e_{i}, e_{j}\right]_{E}=c_{i j}^{k} f_{k} .
$$

Since the algebra is 2-step nilpotent, it satisfies the Jacobi identity and it is a Lie algebra. We can them embed $D$ in $E$ by putting $\varphi\left(e_{i}\right)=\left(e_{i}+f_{i}\right)$ and take the complementary subspace $D^{\perp}$ as spanned by elements $e_{i}-f_{i}$. The projection $P: E \rightarrow D$ is therefore given by

$$
P\left(e_{i}\right)=e_{i}+f_{i}, \quad P\left(f_{i}\right)=e_{i}+f_{i} .
$$

The nonholonomic bracket on the embedded submanifold $D$ is therefore

$$
\left[e_{i}+f_{i}, e_{j}+f_{j}\right]=P\left(c_{i j}^{k} f_{k}\right)=c_{i j}^{k}\left(e_{k}+f_{k}\right),
$$

thus the original bracket in $D$.

For a general quasi-Lie algebroid the situation is much more complicated and we do not know a full characterization of nonholonomic quasi-Lie algebroids. Note however that not all quasi-Lie algebroids are nonholonomic reductions of Lie algebroids.

Example 4.2. Take a quasi-Lie algebroid structure $\varepsilon$ on $D=\mathbf{T} M$ with the anchor $\rho=\operatorname{id}_{\mathbf{T} M}$. $\operatorname{The}$ corresponding tensor on $\mathbf{T}^{*} M$ has therefore the local form

$$
\Pi_{\varepsilon}=\frac{1}{2} c_{i j}^{k}(x) p_{k} \partial_{p_{i}} \wedge \partial_{p_{j}}+\partial_{p_{i}} \wedge \partial_{x^{i}}
$$

Suppose that the corresponding bracket is a nonholonomic reduction of a Lie algebroid bracket $[\cdot, \cdot]_{E}$ of a bigger vector bundle $E$ along a projection $P$,

$$
[X, Y]_{\varepsilon}=P\left([X, Y]_{E}\right) .
$$

Since the anchor $\rho$ of $D$ is the restriction of the anchor $\rho_{E}$ to $D$, the Lie algebroid $E$ satisfies $\rho_{E}\left(E_{\mid M}\right)=$ $\mathbf{T} M$, thus $E_{\mid M}=D \oplus_{M} K$, where $K$ is the kernel of $\rho_{E}$ over $M$. For sections $X, Y$ of $D$ we have therefore $\rho_{E}\left([X, Y]_{E}\right)=\rho([X, Y])$, thus, as the anchor map is for Lie algebroids a homomorphism of brackets,

$$
\rho([X, Y])=\rho_{E}\left([X, Y]_{E}\right)=\left[\rho_{E}(X), \rho_{E}(Y)\right]_{v f}=[\rho(X), \rho(Y)]_{v f},
$$

where $[\cdot, \cdot]_{v f}$ is the bracket of vector fields. We get that the anchor $\rho$ maps the algebroid bracket into the bracket of vector fields which is not the case for generally non-zero structure functions $c_{i j}^{k}(x)$.

\section{Nonholonomic Systems of MechanicAL TYPE}

Let us consider now on our general algebroid $(E, \varepsilon)$ a Lagrangian $L: E \rightarrow \mathbb{R}$ of mechanical type, i.e.

$$
L(e)=\frac{1}{2} G(e, e)-V(\tau(e))
$$

where $\mathcal{G}: E \times_{M} E \rightarrow \mathbb{R}$ is a bundle metric on $E$ and $V: M \rightarrow \mathbb{R}$ is the potential function. Let us consider also a a vector subbundle $D$ of $E$ over $D_{M}$ satisfying the strong admissibility condition. Having the metric $\mathcal{G}$ in $E$, we have the natural decomposition $E_{\mid D_{M}}=D \oplus_{D_{M}} D^{\perp}$ with $D^{\perp}$ being the orthogonal complement of $D$ with respect to the metric $\mathcal{G}$, accompanied with the corresponding (this time - orthogonal) projection $P: E_{\mid D_{M}} \rightarrow D$. The fundamental observation in this case is the following.

Theorem 5.1. The nonholonomic Tulczyjew differential $\Lambda_{L}^{D}$ coincides with the Tulczyjew differential $\Lambda_{l}$ associated with the restricted Lagrangian $l=L_{\mid D}: D \rightarrow \mathbb{R}$ and the nonholonomic algebroid structure $\varepsilon_{P}$ on D. In other words, the nonholonomic Euler-Lagrange equations associated with $L$ and the nonholonomic constraint $D$ in $E$ coincide with the non-constrained Euler-Lagrange equations for the Lagrangian function $l$ on the algebroid $\left(D, \varepsilon_{P}\right)$. 
Proof. Let us consider local coordinates $\left(x^{I}\right)=\left(x^{i}, x^{\iota}\right)$ on a open set $U$ of $M$ such that $D_{M}$ is determined by the constraint $x^{\iota}=0$. Take a local basis of orthonormal sections $\left\{e_{a}, e_{\alpha}\right\}$ of $E$ adapted to the orthogonal decomposition $E_{\mid D_{M}}=D \oplus_{\mid D_{M}} D^{\perp}$, i.e, $\operatorname{span}\left\{e_{a}\right\}=D$ and $\operatorname{span}\left\{e_{\alpha}\right\}=D^{\perp}$. We get the induced coordinates $\left(x^{I}, y^{A}\right)$ in $E$ in which the Lagrangian takes the form

$$
L(x, y)=\frac{1}{2} \sum_{A}\left(y^{A}\right)^{2}-V\left(x^{i}, x^{\iota}\right) .
$$

Note that the 'mass' is 1 in these coordinates. On $E_{\mid D_{M}}$ we have the induced coordinates $\left(x^{i}, y^{a}, y^{\alpha}\right)$, so $D$ is locally determined by the constraints $x^{\iota}=0, y^{\alpha}=0$ and the restricted Lagrangian is:

$$
l\left(x^{i}, y^{a}\right)=\frac{1}{2} \sum_{a=1}^{r}\left(y^{a}\right)^{2}-V\left(x^{i}, 0\right) .
$$

In our coordinates, the projector $P$ reads

$$
P\left(x^{i}, y^{a}, y^{\alpha}\right)=\left(x^{i}, y^{a}\right)
$$

and we deduce that

$$
\varepsilon_{P}\left(x^{i}, y^{a}, p_{j}, \xi_{b}\right)=\left(x^{i}, \xi^{a}, \rho_{d}^{j}\left(x^{i}, 0\right) y^{d}, \mathcal{C}_{d b}^{c}\left(x^{i}, 0\right) y^{d} \xi_{c}+\sigma_{b}^{k}\left(x^{i}, 0\right) p_{k}\right),
$$

so that

$$
\varepsilon_{P} \circ \mathrm{d} l\left(x^{i}, y^{a}\right)=\left(x^{i}, y^{a}, \rho_{d}^{j}\left(x^{i}, 0\right) y^{d}, \sum_{c} \complement_{d b}^{c}\left(x^{i}, 0\right) y^{d} y^{c}-\sigma_{b}^{k} \frac{\partial V}{\partial x^{k}}\left(x^{i}, 0\right)\right) .
$$

We get therefore the Euler-Lagrange equations for the Lagrangian $l$ on $D$ of the form

$$
\begin{aligned}
\frac{d x^{j}}{d t} & =\rho_{a}^{j}\left(x^{i}, 0\right) y^{a}, \\
\frac{d}{d t}\left(\frac{\partial l}{\partial y^{b}}\left(x^{i}, y^{a}\right)\right) & =\mathcal{C}_{d b}^{c}\left(x^{i}, 0\right) y^{d} y^{c}+\sigma_{b}^{j}(x) \frac{\partial l}{\partial x^{j}}\left(x^{i}, y^{a}\right),
\end{aligned}
$$

which are exactly the nonholonomic equations 3.7 and $(3.8)$, if we take to account that

and

$$
\frac{\partial L}{\partial y^{C}}\left(x^{i}, 0, y^{a}, 0\right)=\delta_{a}^{C} y^{a}
$$

$$
\sigma_{b}^{\gamma}\left(x^{i}, 0\right)=0
$$

The above theorem makes it clear that, at least for Lagrangians of mechanical type, the nonholonomic Euler-Lagrange equations are just Euler-Lagrange equations, but on the nonholonomic restriction of the algebroid. In any case, passing to a liner nonholonomic constraint does not move us out of the Mechanics on algebroids. Our theory is therefore complete with respect to passing to the nonholonomic case, what was the main problem in understanding the nonholonomic constraint in the Lie algebroid (thus canonical) case.

Having interpreted the nonholonomic Euler-Lagrange equations as just Euler-Lagrange equations, but on a reduced algebroid, we can use the generalization of Noether Theorem formulated for general algebroids in 9] to obtain a nonholonomic Noether Theorem, at least for Lagrangians of mechanical type.

The generalized Noether Theorem for a general algebroid $(E, \varepsilon)$ is based on the concept of the complete lift $\mathrm{d}_{\top}^{\varepsilon}(K)$ of tensor fields $K$ being sections of the tensor products $E^{\otimes k}$ to the corresponding contravariant tensor fields on $E$. This is a natural generalization of the standard concept of the tangent lift $\mathrm{d}_{\mathrm{T}}$ which lifts contravariant tensors on a manifold $M$ to the corresponding tensor fields on $\mathbf{T} M$, (cf. [11, 37]).

For a vector bundle $\tau: E \rightarrow M$, let $\otimes^{k}(\tau)$ be the space of sections of the tensor-product bundle $E^{\otimes k}$ over $M$. With any tensor field $K \in \otimes^{k}(\tau)$ we can associate the linear function $\iota(K)$ on the dual bundle $\left(E^{\otimes k}\right)^{*}=\left(E^{*}\right)^{\otimes k}$ and the vertical lift $\mathrm{v}_{\tau}(K) \in \otimes^{k}\left(\tau_{E}\right)$ In local coordinates,

$$
\iota\left(f^{a_{1} \cdots a_{k}}(x) e_{a_{1}} \otimes \cdots \otimes e_{a_{k}}\right)=f^{a_{1} \cdots a_{k}}(x) \xi_{a_{1} \cdots a_{k}}
$$


and

$$
\mathrm{v}_{\tau}\left(f^{a_{1} \cdots a_{k}}(x) e_{a_{1}} \otimes \cdots \otimes e_{a_{k}}\right)=f^{a_{1} \cdots a_{k}}(x) \partial_{y^{a_{1}}} \otimes \cdots \otimes \partial_{y^{a_{k}}} .
$$

A particular case of the vertical lift is the lift $\mathrm{v}_{\mathrm{T}}(K)$ of a contravariant tensor field $K$ on $M$ into a contravariant tensor field on $\mathbf{T} M$. It is well known (see [37, 11]) that in the case of $E=\mathbf{T} M$ we have also the tangent lift $\mathrm{d}_{\mathrm{T}}: \otimes\left(\tau_{M}\right) \rightarrow \otimes\left(\tau_{\mathbf{T} M}\right)$ which is a $\mathrm{v}_{\mathrm{T}}$-derivation. What has been done for the tangent bundle, can be repeated in the case of an arbitrary algebroid $(E, \varepsilon)$. Note first that we can extend $\varepsilon$ naturally to mappings (cf. [13, 12])

$$
\varepsilon^{\otimes r}: \otimes_{E}^{r} \mathbf{T}^{*} E \longrightarrow \mathbf{T} \otimes_{M}^{r} E^{*}, \quad r \geq 0 .
$$

Theorem 5.2. 13,12 Let $(E, \varepsilon)$ be an algebroid. For $K \in \otimes^{k}(\tau), k \geq 0$, the equality

$$
\iota\left(\mathrm{d}_{\mathrm{T}}^{\varepsilon}(K)\right)=\mathrm{d}_{\mathbf{T}}(\iota(K)) \circ \varepsilon^{\otimes k}
$$

defines the tensor field $\mathrm{d}_{\mathrm{T}}^{\varepsilon}(K) \in \otimes^{k}\left(\tau_{E}\right)$ which is linear and the mapping

$$
\mathrm{d}_{\mathrm{T}}^{\varepsilon}: \oplus_{k} \otimes^{k}(\tau) \longrightarrow \oplus_{k} \otimes^{k}\left(\tau_{E}\right)
$$

is a $v_{\mathrm{T}}$-derivation of degree 0 . In local coordinates, the lifts of functions on $M$ and sections of $E$ read:

$$
\begin{aligned}
\mathrm{d}_{\mathbf{T}}^{\varepsilon}(f(x)) & =y^{a} \rho_{a}^{i}(x) \frac{\partial f}{\partial x^{i}}(x), \\
\mathrm{d}_{\mathbf{T}}^{\varepsilon}\left(f^{a}(x) e_{a}\right) & =f^{a}(x) \sigma_{a}^{i}(x) \partial_{x^{i}}+\left(y^{a} \rho_{a}^{i}(x) \frac{\partial f^{c}}{\partial x^{i}}(x)+\mathcal{C}_{a b}^{c}(x) y^{a} f^{b}(x)\right) \partial_{y^{c}} .
\end{aligned}
$$

Conversely, if $D: \oplus_{k} \otimes^{k}(\tau) \longrightarrow \oplus_{k} \otimes^{k}\left(\tau_{E}\right)$ is a $v_{\mathrm{T}}$-derivation of degree 0 such that $D(K)$ is linear for each $K \in \otimes^{1}(\tau)$, then there is an algebroid structure $\varepsilon$ on $\tau: E \rightarrow M$ such that $D=\mathrm{d}_{\mathrm{T}}^{\varepsilon}$. This algebroid structure is a Lie algebroid if and only if

$$
\mathrm{d}_{\mathbf{T}}^{\varepsilon}\left([X, Y]_{\varepsilon}\right)=\left[\mathrm{d}_{\mathbf{T}}^{\varepsilon}(X), \mathrm{d}_{\mathbf{T}}^{\varepsilon}(Y)\right]_{v f}
$$

for all $X, Y \in \otimes^{1}(\tau)$.

Suppose now that we are dealing with a Lagrangian $L$ of mechanical type and a nonholonomic constraint subbundle $D$ of $(E, \varepsilon)$ satisfying the strong admissibility assumption, so that we have also the nonoholomic reduced algebroid $\left(D, \varepsilon_{P}\right)$. We will say that a pair $(X, f)$ consisting of a section $X$ of $D$ and a function $f$ on $D_{M}$ is a symmetry of the nonholonomic problem associated with the Lagrangian $L$, if

$$
\mathrm{d}_{\mathrm{T}}^{\varepsilon_{P}}(X)(l)=\mathrm{d}_{\mathrm{T}}^{\varepsilon_{P}}(f),
$$

where $l=L_{\mid D}$ is the restriction of the Lagrangian to the constraint.

Theorem 5.3. [Nonholonomic Noether Theorem] The following are equivalent:

(a) the pair $(X, f)$ is a symmetry of the nonholonomic problem associated with the Lagrangian l;

(b) the function $\iota_{X}-f \circ \tau_{D^{*}}$ on $D^{*}$ is a constant of the motion of the nonholonomic phase dynamics on $D^{*}$, i.e. $\mathrm{d}_{\mathrm{T}}\left(\iota_{X}-f \circ \tau_{D^{*}}\right)$ vanishes on $\Gamma_{L}^{D}$;

(c) the function

$$
\left(\iota_{X}-f \circ \tau_{D^{*}}\right) \circ \lambda_{L}^{D}
$$

is a constant of the motion for the nonholonomic Euler-Lagrange equation.

Proof. The proof is completely analogous to that for Theorem 4 in 9 .

For a section $X$ of $D$, not verifying necessarily condition (5.5), we obtain

$$
\mathrm{d}_{\mathrm{T}}^{\varepsilon_{P}}(X)(l) \circ \gamma(t)=\frac{\mathrm{d}}{\mathrm{d} t}\left(\iota_{X} \circ \lambda_{L}^{D} \circ \gamma(t)\right)
$$

for any solution $\gamma: \mathbb{R} \rightarrow D$ of the nonholonomic Euler-Lagrange equation. This last equation can be interpreted as a general version of the nonholonomic momentum equation studied for several authors (see [7, 2] and references therein) for nonholonomic systems with symmetry. 


\section{EXAMPLES}

Example 6.1. The Chaplygin sleigh.

As an example of nonholonomic system on a Lie algebra, we study the Chaplygin sleigh which describes a rigid body sliding on a plane. The body is supported in three points, two of which slides freely without friction while the third point is a knife edge. This imposes the constraint of no motion orthogonal to this edge (see [5, 28,).

The configuration space before reduction is the Lie group $G=S E(2)$ of the Euclidean motions of the 2-dimensional plane $\mathbb{R}^{2}$. We will need in the sequel to fix some notation about the Lie algebra $\mathfrak{s e}(2)$. First of all its elements are matrices of the form

$$
\hat{\xi}=\left(\begin{array}{ccc}
0 & \xi_{3} & \xi_{1} \\
-\xi_{3} & 0 & \xi_{2} \\
0 & 0 & 0
\end{array}\right)
$$

and a basis of the Lie algebra $\mathfrak{s e}(2) \cong \mathbb{R}^{3}$ is given by

$$
E_{1}=\left(\begin{array}{ccc}
0 & 0 & 1 \\
0 & 0 & 0 \\
0 & 0 & 0
\end{array}\right), \quad E_{2}=\left(\begin{array}{ccc}
0 & 0 & 0 \\
0 & 0 & 1 \\
0 & 0 & 0
\end{array}\right), \quad E_{3}=\left(\begin{array}{ccc}
0 & -1 & 0 \\
1 & 0 & 0 \\
0 & 0 & 0
\end{array}\right) .
$$

We have that

$$
\left[E_{3}, E_{1}\right]=E_{2}, \quad\left[E_{2}, E_{3}\right]=E_{1}, \quad\left[E_{1}, E_{2}\right]=0 .
$$

An element $\xi \in \mathfrak{s e}(2)$ is of the form

$$
\xi=v_{1} E_{1}+v_{2} E_{2}+\omega E_{3} .
$$

The Chaplygin system is described by the kinetic Lagrangian function

$$
\begin{aligned}
\mathfrak{s e}(2) & \longrightarrow \mathbb{R} \\
\left(v_{1}, v_{2}, \omega\right) & \longmapsto \frac{1}{2}\left[\left(J+m\left(a^{2}+b^{2}\right)\right) \omega^{2}+m v_{1}^{2}+m v_{2}^{2}-2 b m \omega v_{1}-2 a m \omega v_{2}\right]
\end{aligned}
$$

where $m$ and $J$ denotes the mass and moment of inertia of the sleigh relative to the contact point and $(a, b)$ represents the position of the center of mass with respect to the body frame determined placing the origin at the contact point and the first coordinate axis in the direction of the knife axis. Additionally, the system is subjected to the nonholonomic constraint determined by the linear subspace of $\mathfrak{s e}(2)$ :

$$
D=\left\{\left(v_{1}, v_{2}, \omega\right) \in \operatorname{se}(2) \mid v_{2}=0\right\} .
$$

Instead of $\left\{E_{1}, E_{2}, E_{3}\right\}$ we take the basis of $\mathfrak{s e}(2)$ :

$$
\left\{e_{1}=E_{3}, e_{2}=E_{1}, e_{3}=-m a E_{3}-m a b E_{1}+\left(J+m a^{2}\right) E_{2}\right\}
$$

which is a basis adapted to the decomposition $D \oplus D^{\perp} ; D=\operatorname{span}\left\{e_{1}, e_{2}\right\}$ and $D^{\perp}=\operatorname{span}\left\{e_{3}\right\}$.

In the induced coordinates $\left(y^{1}, y^{2}\right)$ on $D$ the restricted lagrangian is

$$
l\left(y^{1}, y^{2}\right)=\frac{1}{2}\left[\left(J+m\left(a^{2}+b^{2}\right)\right)\left(y^{1}\right)^{2}+m\left(y^{2}\right)^{2}-2 b m y^{1} y^{2}\right]
$$

and moreover,

$$
\left[e_{1}, e_{2}\right]_{\varepsilon_{P}}=\frac{m a}{J+m a^{2}} e_{1}+\frac{m a b}{J+m a^{2}} e_{2},
$$

Therefore, $\mathcal{C}_{12}^{1}=\frac{m a}{J+m a^{2}}$ and $\mathcal{C}_{12}^{2}=\frac{m a b}{J+m a^{2}}$.

Then,

$$
\left.\varepsilon_{P} \circ \mathrm{d} l\left(y^{1}, y^{2}\right)\right)=\left(\left(J+m\left(a^{2}+b^{2}\right)\right) y^{1}-b m y^{2}, m y^{2}-b m y^{1},-m a y^{1} y^{2}, m a\left(y^{1}\right)^{2}\right) .
$$

In consequence, the equations of motion are:

$$
\begin{aligned}
\left(J+m\left(a^{2}+b^{2}\right)\right) \dot{y}^{1}-b m \dot{y}^{2} & =-m a y^{1} y^{2} \\
m \dot{y}^{2}-b m \dot{y}^{1} & =m a\left(y^{1}\right)^{2}
\end{aligned}
$$


or,

$$
\begin{aligned}
\dot{y}^{1} & =\frac{m a}{J+m a^{2}} y^{1}\left(b y^{1}-y^{2}\right) \\
\dot{y}^{2} & =\frac{m a}{J+m a^{2}} y^{1}\left(\left(J+m\left(a^{2}+b^{2}\right)\right) y^{1}-b y^{2}\right) .
\end{aligned}
$$

Example 6.2. The snakeboard

As a mechanical system the snakeboard has as configuration space $Q=S E(2) \times T^{2}$ with coordinates $(x, y, \theta, \psi, \phi)($ see [3, 18, 17]).

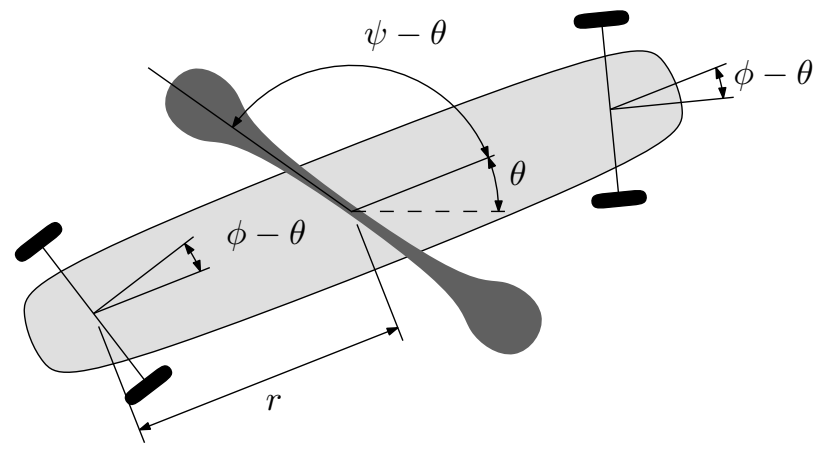

The nonholonomic dynamics is described by

- The Lagrangian

$$
L(q, \dot{q})=\frac{1}{2} m\left(\dot{x}^{2}+\dot{y}^{2}\right)+\frac{1}{2}\left(J+2 J_{1}\right) \dot{\theta}^{2}+\frac{1}{2} J_{0}(\dot{\theta}+\dot{\psi})^{2}+J_{1} \dot{\phi}^{2}
$$

where $m$ is the total mass of the board, $J>0$ is the moment of inertia of the board, $J_{0}>0$ is the moment of inertia of the rotor of the snakeboard mounted on the body's center of mass and $J_{1}>0$ is the moment of inertia of each wheel axles. The distance between the center of the board and the wheels is denoted by $r$. For simplicity (see 17]), we assume that $J+J_{0}+2 J_{1}=m r^{2}$.

- The nonholonomic constraints induced by the non sliding condition in the sideways direction of the wheels:

$$
\begin{aligned}
& -\dot{x} \sin (\theta+\phi)+\dot{y} \cos (\theta+\phi)-r \dot{\theta} \cos \phi=0 \\
& -\dot{x} \sin (\theta-\phi)+\dot{y} \cos (\theta-\phi)+r \dot{\theta} \cos \phi=0 .
\end{aligned}
$$

Observe that the Lagrangian is induced by the riemannian metric $\mathcal{G}$ on $Q$,

$$
\mathcal{G}=m d x^{2}+m d y^{2}+m r^{2} d \theta^{2}+J_{0} d \theta \otimes \psi+J_{0} d \psi \otimes d \theta+J_{0} d \psi^{2}+2 J_{1} d \phi^{2} .
$$

The constraint subbundle $\tau_{D}: D \longmapsto Q$ is

where

$$
D=\operatorname{span}\left\{e_{1}=\frac{\partial}{\partial \psi}, e_{2}=\frac{\partial}{\partial \phi}, e_{3}=a \frac{\partial}{\partial x}+b \frac{\partial}{\partial y}+c \frac{\partial}{\partial \theta}\right\} .
$$

$$
\begin{aligned}
a & =-r(\cos \phi \cos (\theta-\phi)+\cos \phi \cos (\theta+\phi))=-2 r \cos ^{2} \phi \cos \theta \\
b & =-r(\cos \phi \sin (\theta-\phi)+\cos \phi \sin (\theta+\phi))=-2 r \cos ^{2} \phi \sin \theta \\
c & =\sin (2 \phi) .
\end{aligned}
$$

The orthogonal complement of $D$ is spanned by

$$
D^{\perp}=\operatorname{span}\left\{e_{4}=-b \frac{\partial}{\partial x}+a \frac{\partial}{\partial y}, e_{5}=\left(c J-c m r^{2}\right) \frac{\partial}{\partial x}+a m \frac{\partial}{\partial \theta}-a m \frac{\partial}{\partial \psi}\right\} .
$$

In the induced coordinates $\left(x, y, \theta, \psi, \phi, y^{1}, y^{2}, y^{3}\right)$ on $D$ the restricted lagrangian is

$$
l\left(\left(x, y, \theta, \psi, \phi, y^{1}, y^{2}, y^{3}\right)=2 m r^{2} \cos ^{2} \phi\left(y^{3}\right)^{2}+J_{0} c y^{1} y^{3}+\frac{1}{2} J_{0}\left(y^{1}\right)^{2}+J_{1}\left(y^{2}\right)^{2} .\right.
$$


where now the nonholonomic constraints are rewritten as: $y^{4}=0$ and $y^{5}=0$. After some straightforward computations we deduce that

$$
\begin{aligned}
& {\left[e_{1}, e_{2}\right]_{\varepsilon_{P}}=0} \\
& {\left[e_{1}, e_{3}\right]_{\varepsilon_{P}}=0,} \\
& {\left[e_{2}, e_{3}\right]_{\varepsilon_{P}}=\frac{2 m r^{2} \cos ^{2} \phi}{m r^{2}-J_{0} \sin ^{2} \phi} e_{1}-\frac{\left(m r^{2}+\cos 2 \phi\right) \tan \phi}{m r^{2}-J_{0} \sin ^{2} \phi} e_{3} .}
\end{aligned}
$$

Thus, the unique non vanishing structure functions are:

$$
\mathcal{C}_{23}^{1}=-\mathcal{C}_{32}^{1}=\frac{2 m r^{2} \cos ^{2} \phi}{m r^{2}-J_{0} \sin ^{2} \phi}, \quad \mathcal{C}_{23}^{3}=-\mathcal{C}_{32}^{3}=-\frac{\left(m r^{2}+\cos 2 \phi\right) \tan \phi}{m r^{2}-J_{0} \sin ^{2} \phi} .
$$

Therefore, the equation of motion of the snakeboard are

$$
\begin{gathered}
\dot{x}=a y^{3}, \quad \dot{y}=b y^{3}, \quad \dot{\theta}=c y^{3}, \quad \dot{\psi}=y^{1}, \quad \dot{\phi}=y^{2}, \\
\frac{d}{d t}\left(y^{1}+c y^{3}\right)=0, \quad \frac{d}{d t}\left(y^{2}\right)=0, \\
\frac{d}{d t}\left(4 m r^{2} \cos ^{2} \phi y^{3}+J_{0} c y^{1}\right)=2 J_{0} \cos (2 \phi) y^{1} y^{2}-2 m r^{2} \sin (2 \phi) y^{2} y^{3} .
\end{gathered}
$$

\section{REFERENCES}

[1] V. I. Arnold: Dynamical Systems, Vol. III, Springer-Verlag, New York, Heidelberg, Berlin, 1998.

[2] A. M. Bloch, P. S. Krishnaprasad, J. E. Marsden, R. M. Murray: Nonholonomic Mechanical Systems with Symmetry, Arch. Rational Mech. Anal. 136 (1996) 21-99.

[3] F. Bullo, M. Žefran M: On mechanical control systems with nonholonomic constraints and symmetries, Systems \& Control Letters 45 (2) (2002), 133-143.

[4] F. Cantrijn, M. de León and D. Martín de Diego: On almost-Poisson structures in nonholonomic mechanics, Nonlinearity 12 (1999), 721-737.

[5] S. A. Chaplygin: On the theory of the motion of nonholonomic systems. Theorem on the reducing multiplier, Math. Sbornik 28 (2) (1911), 303-314.

[6] J. Cortés, M. de León, J. C. Marrero, D. Martín de Diego, E. Martínez: A survey on Lagrangian Mechanics and control on Lie algebroids and groupoids. International Journal of Geometric Methods in Modern Physics 3 (2006) 509-558.

[7] J. Cortés, M. de León, J. C. Marrero, E. Martínez: Nonholonomic Lagrangian systems on Lie algebroids, Preprint (2005), math-ph/0512003 to appear in Discrete Continuous Dynamical Systems, Serie A.

[8] K. Grabowska, J. Grabowski: Variational calculus with constraints on general algebroids, J. Phys. A: Math. Theor. 41 (2008) 175204

[9] K. Grabowska, J. Grabowski, P. Urbański: Geometrical mechanics on algebroids. Int. J. Geom. Methods Mod. Phys. 3 (3) (2006), 559-575.

[10] J. Grabowski, M. Rotkiewicz: Higher vector bundles and multi-graded symplectic manifolds, preprint, arxiv: math.DG/0702772

[11] J. Grabowski and P. Urbański: Tangent lifts of Poisson and related structures, J. Phys. A 28 (1995), 6743-6777.

[12] J. Grabowski and P. Urbański: Algebroids - general differential calculi on vector bundles, J. Geom. Phys. 31 (1999), $111-141$.

[13] J. Grabowski and P. Urbański: Lie algebroids and Poisson-Nijenhuis structures, Rep. Math. Phys. 40 (1997), $195-208$.

[14] J. Grabowski and P. Urbański: Algebroids - general differential calculi on vector bundles, J. Geom. Phys. 31 (1999), $111-141$

[15] A. Ibort, M. de León, J. C. Marrero and D. Martín de Diego: Dirac brackets in constrained dynamics, Fortschr. Phys. 47 (1999), 459-492.

[16] K. Konieczna and P. Urbański: Double vector bundles and duality, Arch. Math. (Brno) 35 (1999), 59-95.

[17] W.S. Koon, J.E. Marsden: Optimal Control for Holonomic and Nonholonomic Mechanical Systems with Symmetry and Lagrangian Reduction, SIAM Journal on Control and Optimization 35 (1997) 901-929.

[18] M. de León, J. C. Marrero, D. Martín de Diego: Linear almost Poisson structures and Hamilton-Jacobi theory. Applications to nonholonomic Mechanics, Preprint arXiv:0801.4358v1 [math-ph] (2008)

[19] M. de León, J. C. Marrero, E. Martínez: Lagrangian submanifolds and dynamics on Lie algebroids, J. Phys. A Math. Gen. 38 (2005) R241-R308.

[20] P. Libermann: Lie algebroids and mechanics, Arch. Math. (Brno) 32 (1996), 147-162.

[21] K. C. H. Mackenzie: General theory of Lie groupoids and Lie algebroids, London Mathematical Society Lecture Note Series, 213. Cambridge University Press, Cambridge, 2005.

[22] C.-M. Marle: Various approaches to conservative and nonconservative nonholonomic systems, Rep. Math. Phys. 42 (1998), 211-229.

[23] G. Marmo, G. Mendella, W. M. Tulczyjew: Integrability of implicit differential equations. J. Phys. A: Math. Gen. 30 (1), (1995), 149-163 
[24] G. Marmo, G. Mendella, W. M. Tulczyjew: Constrained Hamiltonian systems as implicit differential equations. J. Phys. A: Math. Gen. 30 (1), (1997) 277-293.

[25] E. Martínez: Lagrangian Mechanics on Lie Algebroids, Acta Appl. Math. 67 (2001), 295-320.

[26] E. Martínez: Geometric formulation of Mechanics on Lie algebroids, In Proceedings of the VIII Fall Workshop on Geometry and Physics, Medina del Campo, 1999, Publicaciones de la RSME, 2 (2001), 209-222.

[27] E. Martínez: Variational calculus on Lie algebroids, ESAIM: Control, Optimisation and Calculus of Variations 14 (2008), 356 .

[28] J. Neimark and N. Fufaev: Dynamics of Nonholonomic Systems, Translation of Mathematics Monographs, 33, AMS, Providence, RI, 1972

[29] W. Tulczyjew: Les sous-variétés lagrangiennes et la dynamique hamiltonienne, C.R. Acad. Sci. Paris 283 (1976), 15-18.

[30] W. Tulczyjew: Les sous-variétés lagrangiennes et la dynamique lagrangienne, C.R. Acad. Sci. Paris 283 (1976), 675-678.

[31] W. Tulczyjew: A note on holonomic constraint, Boston Stud. Philos. Sci. 234 (2003), $403-419$.

[32] W.M. Tulczyjew and P. Urbański: A slow and careful Legendre transformation for singular Lagrangians, The Infeld Centennial Meeting (Warsaw, 1998), Acta Phys. Polon. B 30, (1999), 2909-2978.

[33] J. Pradines: Fibrés vectoriels doubles et calcul des jets non holonomes (French), Notes polycopiées, Amiens, 1974.

[34] J. Pradines: Représentation des jets non holonomes par des morphismes vectoriels doubles soudés (French), $C$. $R$. Acad. Sci. Paris Sér. A $\mathbf{2 7 8}$ (1974), 1523-1526.

[35] A. J. van der Schaft and B. Maschke: On the hamiltonian formulation of nonholonomic machanical systems, Rep. Math. Phys., 34 (1994), 225-233.

[36] A. Weinstein: Lagrangian Mechanics and groupoids, Fields Inst. Comm. 7 (1996), $207-231$.

[37] K. Yano and S. Ishihara: Tangent and Cotangent Bundles, Marcel Dekker, Inc., New York, (1973).

Janusz Grabowski: Polish Academy of Sciences, Institute of Mathematics, Śniadeckich 8, P.O. Box 21, 00-956 WarszaWa, Poland

E-mail address: jagrab@impan.gov.pl

Manuel de León: Instituto de Ciencias Matemáticas (CSIC-UAM-UC3M-UCM), Serrano 123,28006 MADRID, SPAIN

E-mail address: mdeleon@imaff.cfmac.csic.es

Juan Carlos Marrero: Departamento de Matemática Fundamental y Unidad Asociada UlL-CSiC Geometría Diferencial y Mecánica Geométrica, Facultad de Matemáticas, Universidad de la Laguna, La Laguna, Tenerife, Canary Islands, Spain

E-mail address: jcmarrer@ull.es

David Martín de Diego: Instituto de Ciencias Matemáticas (CSiC-UAM-UC3M-UCM), Serrano 123, 28006 MADRID, SPAIN

E-mail address: d.martin@imaff.cfmac.csic.es 